\title{
Inertial Particles in Homogeneous Shear Turbulence: Experiments and Direct Numerical Simulation
}

\author{
Claudia Nicolai • Boris Jacob • \\ Paolo Gualtieri · Renzo Piva
}

Received: 9 January 2013 / Accepted: 30 July 2013

(C) Springer Science+Business Media Dordrecht 2013

\begin{abstract}
The properties of the transport of heavy inertial particles in a uniformly sheared turbulent flow have been investigated by combining experimental and numerical data at particle Stokes number $S t \approx 0.3 \div 0.5$ respectively. As in isotropic turbulence, particles are observed to avoid zones of intense enstrophy and to cluster in strain-dominated regions, resulting in highly intermittent spatial distributions. Moreover, the anisotropy of the mean flow is found to imprint a clear preferential orientation of the particle clusters in the direction of the maximum mean strain. These features are observed both in the numerics and in the experiments, and have been consistently quantified by a number of complementary statistical tools, such as the Voronoï tessellations and the pair correlation function. The latter quantity has been generalized in the form of the Angular Distribution Function and has allowed to evaluate the anisotropy content of the particle field at each scale. The behavior of this observable exhibits the same trend in the two datasets and suggests that, owing to increased inertia, the particle distribution starts to recover isotropy at scales smaller than the carrier velocity field. A proper rescaling of the two datasets in terms of their respective values of the shear scale allows to account for differences in the Reynolds number of experiments and numerics in the range of scales dominated by the mean shear.
\end{abstract}

Keywords Particle-laden shear flow - Anisotropic clustering

C. Nicolai $(\bowtie) \cdot$ P. Gualtieri · R. Piva

Dipartimento di Ingegneria Meccanica e Aerospaziale (DIMA),

Sapienza University, Via Eudossiana 18, 00184 Rome, Italy

e-mail: claudia.nicolai@uniroma1.it

B. Jacob · C. Nicolai

CNR-INSEAN, Marine Technology Research Institute,

Via di Vallerano 139, 00128 Rome, Italy 


\section{Introduction}

The interaction between turbulence and a dispersed phase has been studied for a long time, being of interest for a large number of natural phenomena and technical applications as diverse as the formation of raindrops in clouds, transport of air bubbles in the oceans or injection processes in combustion engines. The most striking feature of this interaction consists in the phenomenon of preferential accumulation. Because of the different inertia between the two phases, particles do not sample the fluid domain uniformly under the action of turbulent fluctuations. In fact, a large number of numerical and experimental studies have shown that small heavy particles are ejected from high-vorticity zones and accumulate in regions of high strain. Lighterthan-fluid particles display the opposite tendency and migrate towards high-vorticity regions (see e.g. the recent Reference [1] which summarizes the relevant work in this area). The non-uniformities which arise in the instantaneous concentration field of both heavy and light particles are of interest per se, since they affect important quantities such as the rate of inter-particle collisions or the velocity of coalescence for bubbles. Also, large peaks of the concentration can be created locally through the mechanism of inertial clustering even at very dilute particles loading, possibly resulting in significant feedback on the carrier field with a profound modification of the turbulence structure even at the largest scales (see e.g. [2] for the case of bubbles dispersed in a boundary layer and [3] for particle laden shear flows).

In this paper, we focus on the interaction between heavy point-like particles and homogeneously sheared turbulence. This prototypical flow is characterized by homogeneous turbulent fluctuations superimposed on a linear mean velocity profile. It is therefore of great importance, since it retains the most relevant feature of a large number of flows of interest for applications, namely, the mean shear. At the same time, however, it does not involve additional complicacies such as those associated with the presence of solid boundaries or spatial inhomogeneities.

We wish to gain insight into the structure and the statistical properties of inertial particle transport in these conditions by combining experimental and numerical data analyzed with a number of statistical tools. In fact, on the one hand the use of numerics gives access to deeper information. On the other hand the interpretation of the numerical results requires some caution, since severe approximations are used when modeling the dispersed phase as an ensemble of point-like particles [1]. Hence, the combined use of experimental and numerical data is advisable for a better understanding of the relevant physical aspects.

\section{Experimental and Numerical Datasets}

As anticipated in the introduction, our investigation relies on two complementary datasets. Actually, when dealing with a complicated experiment such as the present particle-laden homogeneous shear flow, the combined exploitation of experimental and numerical configurations can provide new elements for a better understanding and validation of the results. For instance, as illustrated in Section 3.3 at the end of the paper, one can use DNS data to identify the downstream location of the measurement domain where the dispersed phase has reached a spatially homogeneous state 
Fig. 1 Sketch of the experimental setup

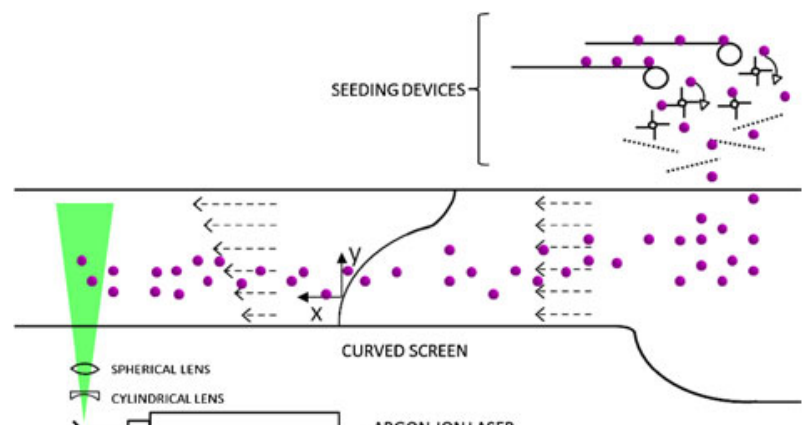

ARGON-ION LASER

and particles have almost lost memory of the large scale inhomogeneities which are unavoidably introduced by the seeding device.

The experimental dataset has been obtained in a laboratory, where a homogeneous shear flow was generated in the central part of a recirculating water channel following the technique described in [4]. In this case, the uniform velocity gradient $S=\partial U / \partial y$ is established downstream of a suitably designed curved screen of uniform solidity, which is placed immediately after the contraction of the water channel, see the sketch in Fig. 1. The main characteristics of the experiment are listed in Table 1. The relevant flow parameters (measured in absence of particles by means of a two-component back-scatter LDV system) are the Reynolds number based on the Taylor micro-scale, $R e_{\lambda} \approx 540$, and the shear parameter $S^{*}$ (see [5]) representative of the intensity of the mean velocity gradient, which is defined as: $S^{*}=S u^{\prime 2} / \epsilon \approx 4.5$. The ratio between the so-called shear scale $L_{S}=\sqrt{\epsilon / S^{3}}$ and the Kolmogorov scale is $L_{S} / \eta \simeq 350$, and provides an indication of the extent of turbulent scales (below $L_{S}$ ) where the velocity field is characterized by an isotropy-recovering dynamics. The experiment has been conducted with spherical glass of density $\rho_{p}=2600 \mathrm{~kg} / \mathrm{m}^{3}$ at very low volumetric concentration $\left(C_{v} \approx 10^{-6}\right)$. A snapshot of the glass beads is given in Fig. 2, where the histogram of particle sizes is also reported. It appears that the distribution of particle diameters is approximately normal, with a mean
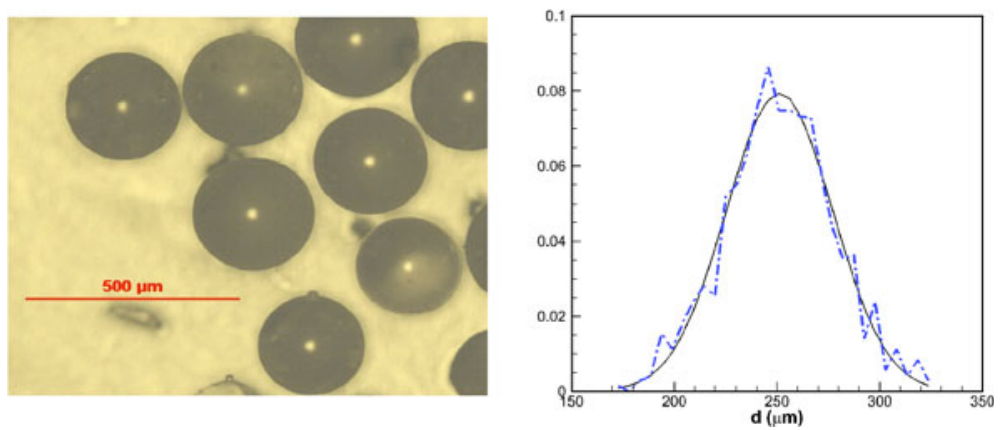

Fig. 2 Left panel: a snapshot of the glass beads acquired with a microscope (50X magnification). Right panel: the pdf of particle diameters (dashed line) as compared to a Gaussian fit (solid line) 
Table 1 The main parameters defining carrier turbulence and solid phase in both the experimental and the numerical datasets

\begin{tabular}{lllllllll}
\hline & $R e_{\lambda}$ & $S^{*}$ & $\eta$ & $L_{S}$ & St & $\Delta r_{p}$ & $N_{p}$ & $\delta$ \\
\hline DNS & 100 & 7 & $0.02\left(^{*}\right)$ & $35 \eta$ & 0.5 & $\sim 10 \eta$ & $\mathcal{O}\left(10^{3}\right)$ & $2 \eta$ \\
EXP & 540 & 4.5 & $200 \mu m$ & $350 \eta$ & 0.28 & $\sim 12 \eta$ & $\mathcal{O}\left(10^{3}\right)$ & $(2 \div 3) \eta$ \\
\hline
\end{tabular}

In order to characterize homogeneously sheared turbulence, two parameters (e.g., the Reynolds number $R e_{\lambda}$ and the shear parameter $S^{*}$ ) are required. The Reynolds number is based on Taylor micro-scale and turbulent intensity $\left(R e_{\lambda}=u^{\prime} \lambda / \nu\right)$ while the shear parameter is defined as $S^{*}=$ $\left(L / L_{S}\right)^{2 / 3}$, where $\eta$ denotes the Kolmogorov scale and $L_{S}$ is the so-called shear scale $L_{S}=\sqrt{\epsilon / S^{3}}$. The particle Stokes number is defined as usual as $S t=\tau_{p} / \tau_{\eta}$, with $\tau_{p}=\rho_{p} d^{2} / 18 \mu_{f}$ and $\tau_{\eta}=\sqrt{\nu_{f} / \epsilon}$. Finally, $\Delta r_{p}$ indicates the mean particle distance evaluated on two dimensional slices of thickness $\delta, N_{p}$ is the mean number of particles per slice and $\delta$ indicates the slice thickness. $(*)$ denotes computational units

value $d_{p} \approx 0.25 \mathrm{~mm}$ (i.e., particles are comparable in size with the Kolmogorov scale $\eta, d_{p} / \eta \approx 1.2$ ) and with a pretty tight standard deviation $\sigma_{d} \approx 25 \mu \mathrm{m}$. The particle Stokes number $S t$, i.e. the ratio of the particle relaxation time to the dissipative timescale, is therefore $S t \approx 0.3$. The instantaneous concentration field has been measured in a longitudinal plane by imaging with a $2848 \mathrm{px} \times 4288 \mathrm{px}$ CCD camera the positions of particles illuminated by a continuous laser sheet. The size of the measurement domain is approximately $150 \times 100 \mathrm{~mm}^{2}$ (with sub-millimetric thickness). The dataset to be discussed in the following consists of 5000 uncorrelated images acquired at a sampling frequency of $2 \mathrm{~Hz}$. Note that the measurement location has been selected at sufficiently large downstream distances from the curved grid so that spurious effects related to the seeding mechanism are ruled out. This aspect has been carefully addressed in order to isolate inertial clustering features from the inhomogeneities introduced by the seeding procedure (see Ref. [6] as well as Section 3.3 for more details). The second dataset is obtained by Direct Numerical Simulations, in which the one-way coupling between solid particles has been implemented using the point-particle approach $[7,8]$. The turbulent parameters are in this case given by $R e_{\lambda} \approx 100$ and $S^{*} \approx 7$ (see Table 1 ), while the ratio of the shear- to the Kolmogorov scale is equal to $L_{s} / \eta \simeq 35$. The Navier-Stokes equations are integrated in a $4 \pi \times 2 \pi \times 2 \pi$ periodic box with a resolution of $256 \times 256 \times 128$ Fourier modes, corresponding to $384 \times 384 \times 192$ collocation points in physical space due to the $3 / 2$ dealiasing rule. The Kolmogorov scale corresponds to $\eta=0.02$. The dispersed phase consists of $N=3 \times 10^{5}$ point-like spherical particles with Stokes number $S t=0.5$, which are initialized with random and homogeneous positions and velocities which are matching that of the local fluid. The only force considered in the numerical simulation is the Stokes drag and the particles evolve according to the equations $\dot{x}_{i}^{p}=v_{i}^{p} ; \quad \dot{v}_{i}^{p}=\tau_{p}^{-1}\left[u_{i}\left(x^{p}, t\right)-v_{i}^{p}(t)\right]$ where $u_{i}\left(x^{p}, t\right)$ is the instantaneous fluid velocity evaluated at the particle position $x_{i}^{p}(t)$ and $\tau_{p}=\rho_{p} d_{p}^{2} /\left(18 v \rho_{f}\right)$ is the Stokes relaxation time. The fluid velocity at the particle position is evaluated by a tri-linear interpolation of the fluid velocity at the closest grid points.

\section{Results}

Typical snapshots of the particle field extracted from the DNS dataset are shown in Fig. 3, where the position of particles belonging to thin slices parallel to the 

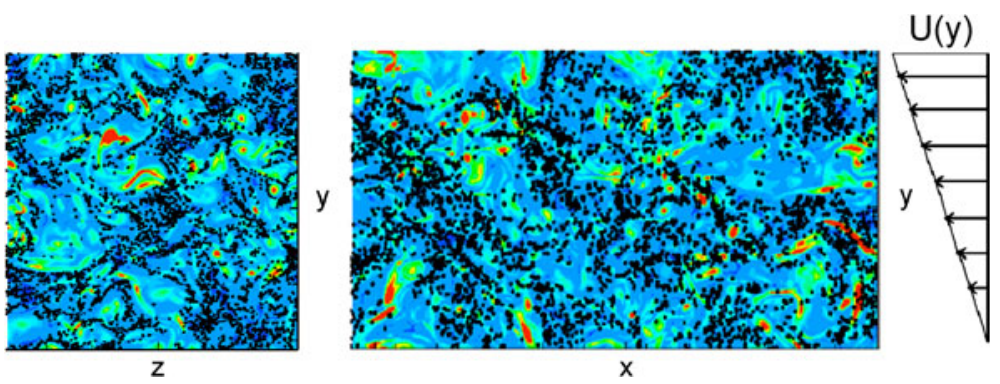

Fig. 3 A typical instantaneous particle distribution extracted from the DNS data superimposed on the vorticity field (high vorticity values correspond to red levels). Left panel: cross-stream plane. Right panel: longitudinal plane

coordinate planes are depicted. The left panel (which refers to a cross-sectional plane) clearly shows that particles avoid zones of intense enstrophy and concentrate in fluid regions of large strain. Zones of large enstrophy correspond to the cores of the large-scale vortices which dominate the dynamics of sheared turbulence. These vortices are known to preferentially align along the direction of maximum mean strain i.e, they are inclined at an average angle of $45^{\circ}$ with respect to the streamwise direction. The signature of these structures is clearly visible in the right panel of Fig. 3, which reports a cut of the computational domain along a longitudinal plane. Consistently with the centrifuging mechanism, particles are observed to aggregate into thin filamentary patches located on the periphery of the large-scale structures. Note that the filamentary structure in which particles aggregate is also apparent in the fields extracted from the experimental dataset, as illustrated in the left panel of Fig. 5, where a typical instantaneous field in the measurement domain is reported together with its companion Voronoï tessellation (which is going to be discussed in the following Section).

From a more quantitative point of view, the correlation between particle concentration peaks and regions of low enstrophy $\left(\omega^{2}\right)$ can be examined in DNS data by recording the value of the enstrophy field at each particle position for several time configurations, and by successively measuring the number of particles associated to each enstrophy level. The plot shown in the left panel of Fig. 4 reports the results concerning two different classes of particles dispersed by the same velocity field (the Stokes numbers are respectively equal to 0.1 and 0.5 ). Particles with higher inertia exhibit comparatively larger concentration in the range of low-enstrophy values, i.e. a larger tendency to concentrate in regions of low enstrophy. This result is corroborated by the plot reported in the right panel of Fig. 4, which compares the probability density function of the enstrophy sampled at particles positions with the pdf of the same quantity measured over the whole fluid domain.

In order to gain further insight into the dispersed phase distribution, a number of appropriate methods are available in the literature. A recent review of these techniques can be found e.g. in Ref. [9]. Among the possible approaches, we choose here two complementary tools, namely the Voronoï decomposition and the analysis based on the Angular Distribution Function. The first approach is particularly useful for extracting the geometrical properties of the particle field, for instance to identify 

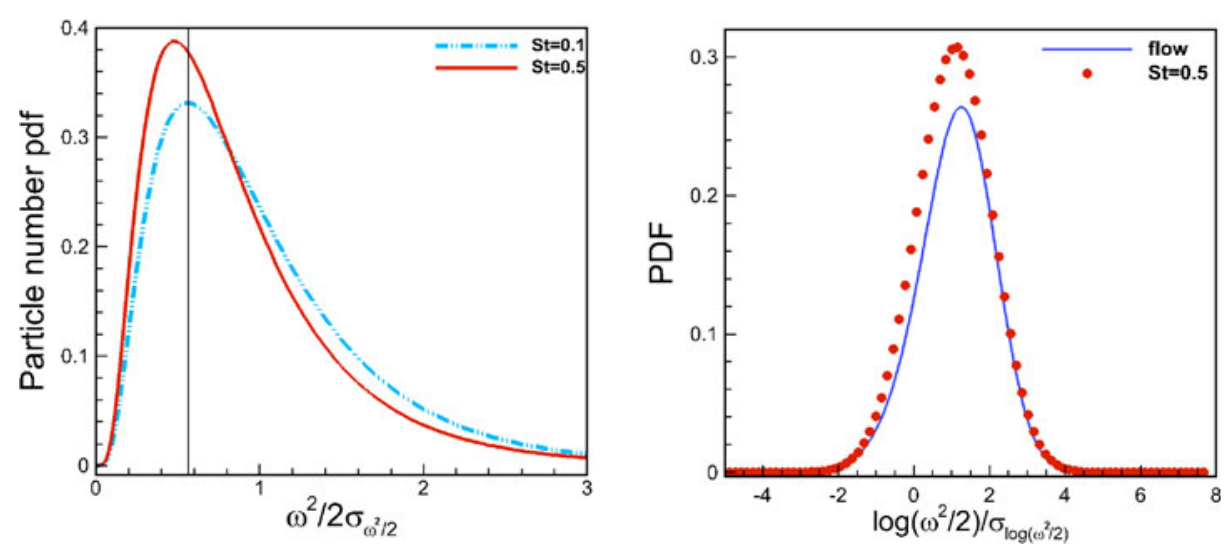

Fig. 4 Left panel: the comparison of the mean particle concentration of inertial particles (respectively with $S t=0.1$ and $S t=0.5$ ) found at different enstrophy levels of the carrier fluid. Right panel: the comparison between the pdf of the enstrophy measured respectively over the whole fluid domain (solid line) and sampled at particle positions only (symbols)

clusters and voids. The second technique is more suitable to identify at each scale the effects induced by the large-scale anisotropy on the particle distribution.

\subsection{Voronoï analysis}

The Voronoï analysis is a technique recently introduced to identify the geometrical properties of particle clusters in isotropic turbulence [10]. For a given set of particles, the Voronoï diagram consists in a space decomposition which associates a cell to each particle center (say, P) in such a way that all points within the cell are closer to $\mathrm{P}$ than to any other particle. For two-dimensional cases such as those considered here, the cell associated to $\mathrm{P}$ is a polygon which is built with the perpendicular bisectors method, i.e., by first detecting the $n$ nearest neighbors $\mathrm{Q}$ of the particle $\mathrm{P}$ and by successively bisecting each segment QP with a line perpendicular to
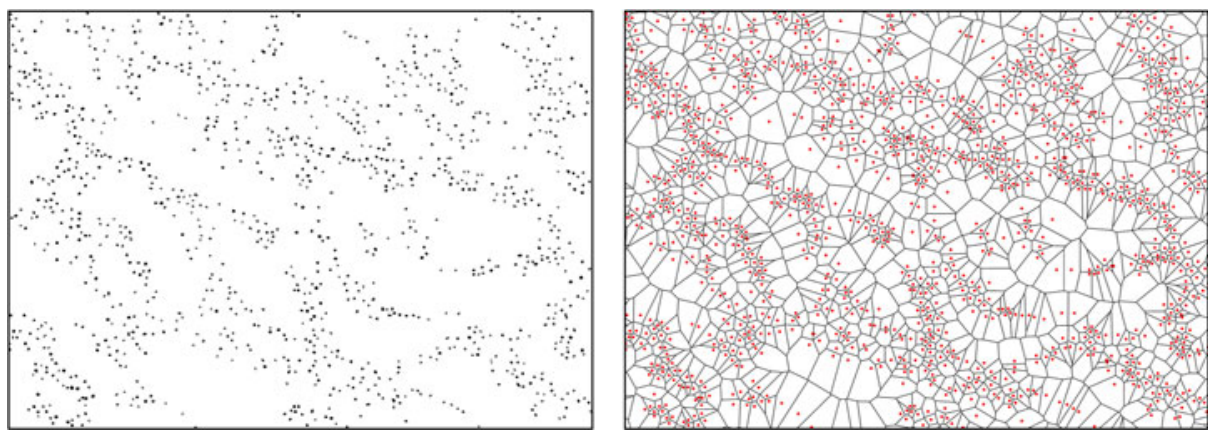

Fig. 5 Left panel: portion of a typical raw image (of size $2340 p x \times 1800 p x$ ) acquired by the CCD camera, showing the instantaneous positions of the dispersed particles. Right panel: the associated Voronoï diagram 

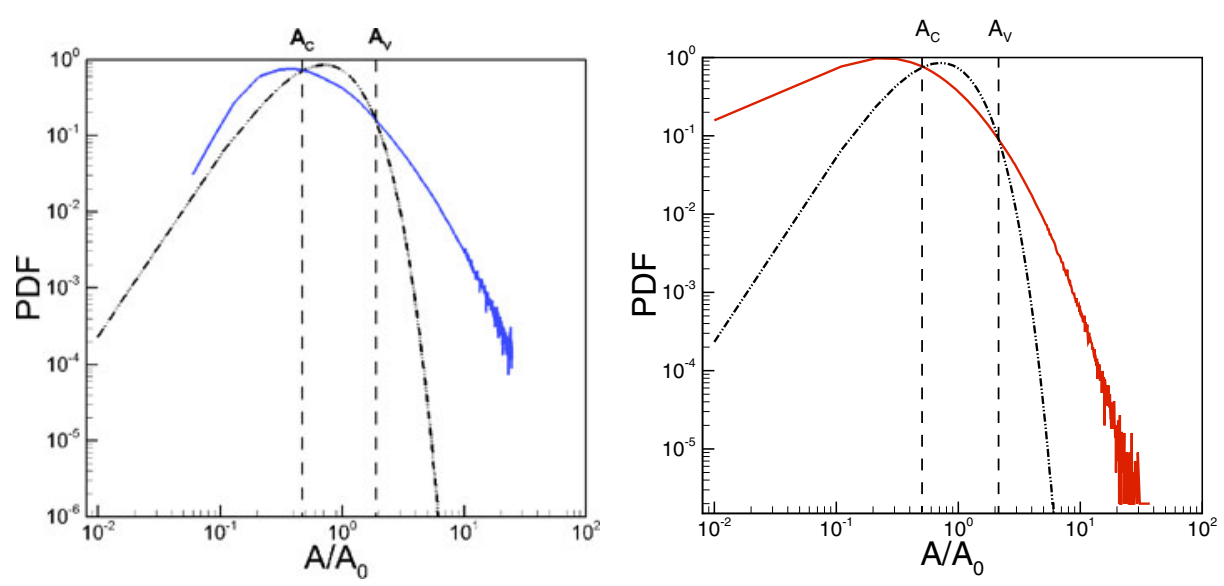

Fig. 6 Left panel: the PDF of the Voronoï areas computed for the experimental dataset (blue line). Right panel: the PDF of the Voronoï areas for the numerical data set (red line). In both panels the black line indicates the PDF of a purely random process at identical mean concentration $1 / A_{0}$

it. The cell associated to $\mathrm{P}$ is given by the region which is enclosed by such $n$ perpendicular bisecting segments. A representative Voronoï tessellation performed on instantaneous 2-D particle fields obtained from the experimental investigation is shown in Fig. 5 (right panel). From the earlier discussion, it follows the important property that the areas of the cells associated to the tessellation are inversely proportional to the local particle concentration, so that the description of the clustering process can be equivalently obtained in terms of statistics of such areas. A possible procedure consists in computing the Probability Distribution Function (PDF) of the cell areas $A$, and comparing it with the distribution pertaining to a purely random process, where an equal amount of particles is distributed over the same region in a fully uncorrelated way. In two dimensions $(d=2)$, the statistics of a purely random process is accurately reproduced by the expression proposed in Ref. [11] in terms of a gamma function, namely the PDF of the normalized Voronoï areas $A / A_{0}$ can be modeled as:

$$
\operatorname{PDF}\left(\frac{A}{A_{0}}\right)=\frac{((3 d+1) / 2)^{(3 d+1) / 2}}{\Gamma((3 d+1) / 2)}\left(\frac{A}{A_{0}}\right)^{(3 d+1) / 2} \exp \left(-\frac{3 d+1}{2}\left(\frac{A}{A_{0}}\right)\right),
$$

where $A_{0}$ is given by $A_{0}=1 / C_{0}$ and $C_{0}$ is the average particle concentration. The PDF of the normalized Voronoï areas $A$ of the two datasets is displayed in Fig. 6 together with the reference distributions corresponding to random processes at identical concentration $C_{0}$.

The comparison of the curves in Figs. 6 and 7 (left column) confirms that the particle distribution for the actual turbulent flow is much more intermittent than expected on a purely random basis, i. e., both very small and very large values of the Voronoï areas occur more frequently. Figures 6 and 7 also show that the behavior is qualitatively similar for both the experimental and the numerical datasets, as 

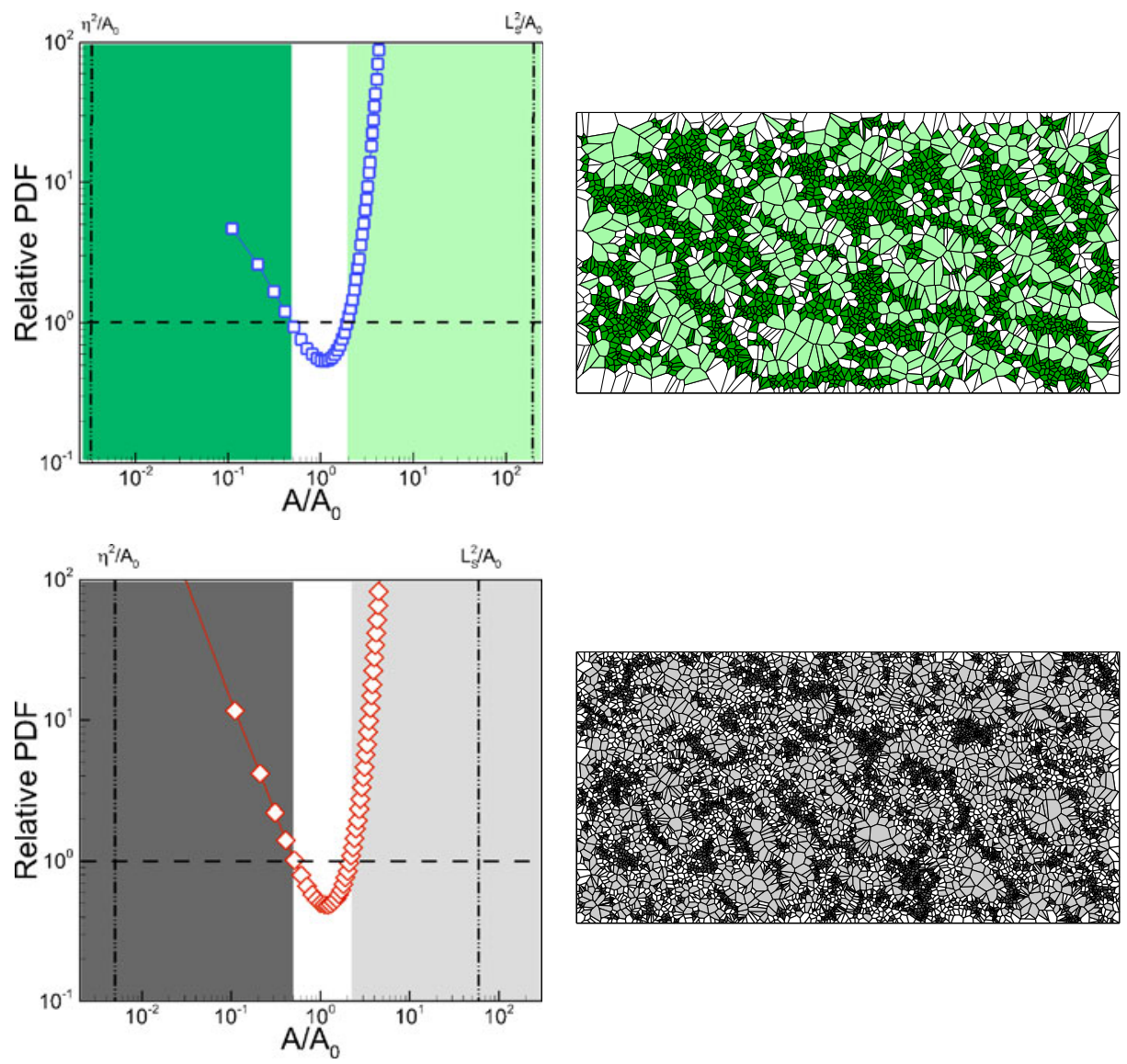

Fig. 7 Left panels: the ratio of the PDF of normalized areas to the PDF of a Poissonian process at identical mean concentration. Right panels: visualization of clustering regions (dark regions) and voids (light regions) obtained from the area connection process. Experimental and numerical data are reported in the top and bottom panels, respectively

confirmed by the good agreement of the standard deviations of the two PDF's, which are respectively equal to $\sigma_{\text {exp }}=0.79 \pm 0.05$ and $\sigma_{D N S}=0.83 \pm 0.04$. The error bar of the experimental result accounts for the statistical dispersion as well as for uncertainties related with the particle identification. The error bar of the numerical result, on the contrary, mostly reflects the variation in the value of $\sigma$ which is observed when the thickness $\delta / \eta$ of the two-dimensional cut is changed between $\delta / \eta=1$ and $\delta / \eta=4$, i.e. in a range of thickness comparable with the experimental situation (the value of $\sigma$ decreases monotonically when $\delta$ is reduced, see Ref. [12] for a thorough discussion of the possible sources of uncertainty affecting its estimate).

To further characterize the statistics of the particle distribution in shear turbulence, it is of value to assess the log-normality of the pdf of Voronoi areas, as this trend has been consistently observed in experimental and numerical simulations of isotropic turbulence [10]. Indeed, such behavior has been confirmed by the analysis of the compressibility features of particle patches by using the alternative approach 

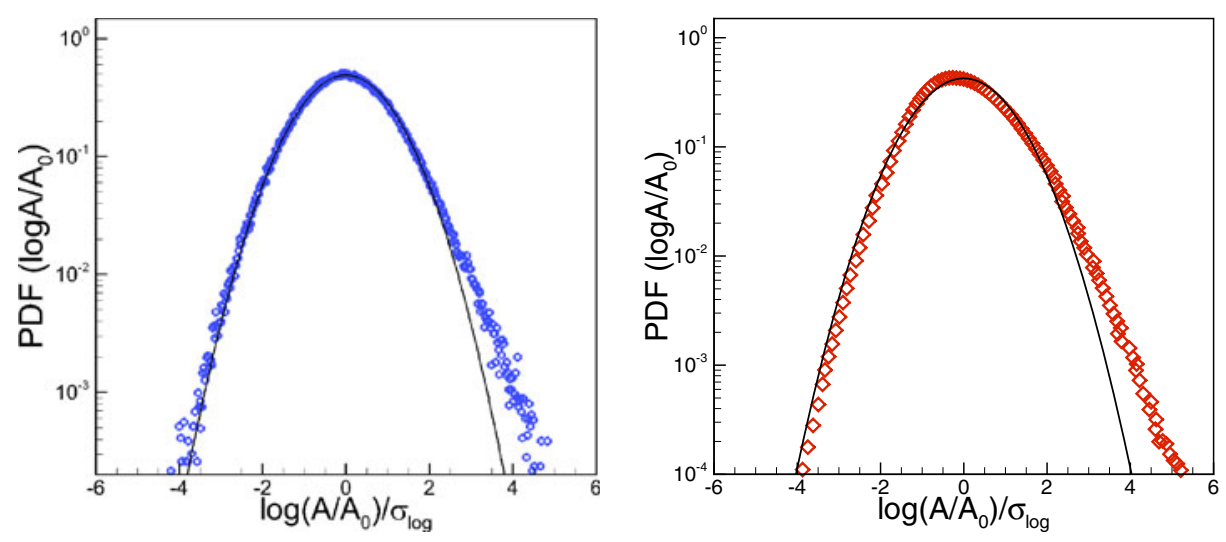

Fig. 8 The comparison between the pdf of the logarithm of normalized Voronoï areas with the corresponding fit based on a Gaussian law. Left panel: experimental dataset. Right panel: DNS dataset

of the Full Lagrangian Method in simulations where particles are transported by homogeneous and isotropic turbulence [13] or by random flows [14]. The test of log-normality is provided in Fig. 8, where the PDF's of the normalized Voronoï areas measured in the experiment and in the numerics are compared with the corresponding Gaussian fits (left and right panel, respectively). At small scales, where the homogeneous shear flow behaves essentially as isotropic turbulence, the data are reasonably well described by a log-normal distribution, in agreement with previous results, see e.g. [10,13]. Departures from log-normality are mostly observed at the large scales, i.e., when the observation scale is comparable with the shear scale $\left(A \geq L_{S}^{2}\right.$ ). This result is not unexpected, and is likely to be ascribed to the different properties of the large-scale structures which are typical of shear-driven turbulence. Note in any case that the deviations from the log-normal behavior are relatively small and confined to large values of $\tilde{A}$, legitimating the use of $\sigma$ as a global measure of the departure from the purely random behavior (as done in isotropic turbulence [12]).

The Voronoï decomposition provides important information on the range of scales involved in the particle dispersion process, i.e. on the characteristic dimension of the regions which may be identified as clusters and voids respectively. In fact, the comparison of the actual PDF of Voronoï areas with its synthetic counterpart naturally introduces two typical dimensions, which are labeled as $A_{C}$ and $A_{V}$ in Fig. 6. Cells with areas $A<A_{C}$ correspond to particle-rich regions, and adjacent cells which fulfill this conditions can be connected by means of region-growing algorithms to form particle clusters. Conversely, adjacent Voronoï polygons with $A>A_{V}$ (representing zones free from particles) can be merged to yield voids. These structures are represented by the dark and light regions in the right panel of Fig. 7. Further insight into the geometrical properties of the connected clustered regions can be gained from the statistical analysis of the associated areas $A_{\text {cluster }}$.

The PDF of such areas is reported in Fig. 9 for both the experimental and the numerical case. Again, favorable agreement between the two datasets is obtained despite the differences in the flow parameters. In particular, both PDF's are found to follow a power-law with an exponent close to -2 in the range of smaller areas 

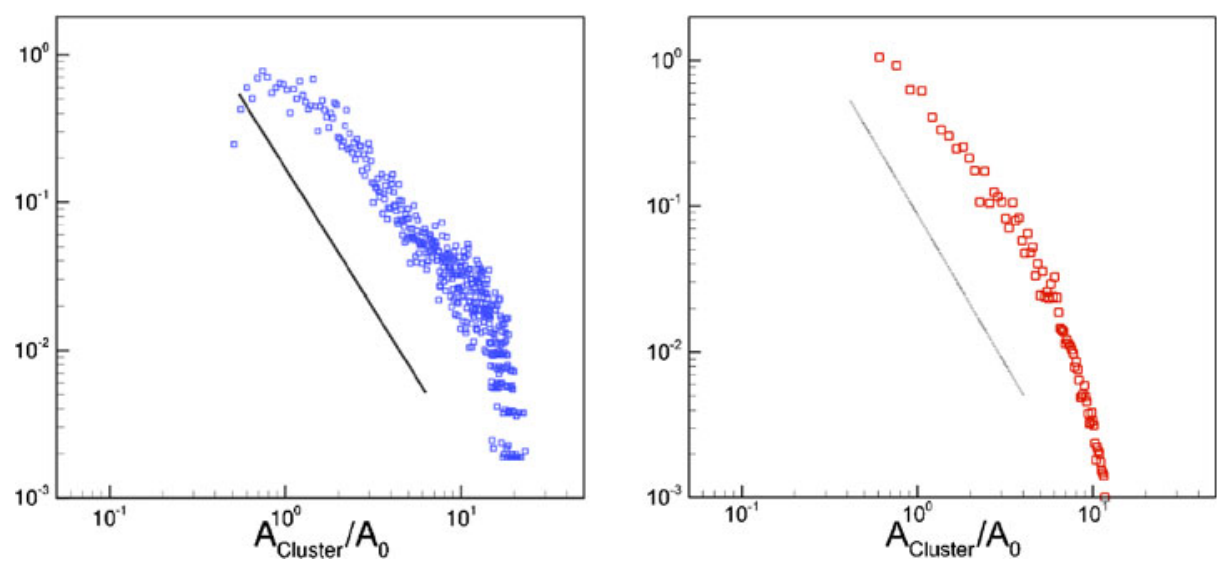

Fig. 9 The PDF of clustering regions computed for both the experimental and numerical cases

where the effect of the mean shear is expected to be less important. Interestingly, this behavior has been already observed in numerical and experimental investigations of particle-laden isotropic turbulence [9], and reveals the important (and possibly universal) self-similar character of the preferential accumulation at small scales.

\subsection{Pair correlation function}

Another technique commonly employed to quantify inertial particle clustering in isotropic turbulence is represented by the so-called Radial Distribution Function (RDF), which measures the probability of finding particle pairs at a given distance $r$ $[15,16]$. In anisotropic flows, a more complete characterization of the particle field need to be performed by retaining the spatial directionality of the statistics. This can be done by means of the Angular Distribution Function (ADF) recently adopted in Ref. [8] and [6] to describe the geometry of particle clusters in presence of a mean shear. The ADF simply gauges the probability of detecting two particles at separation $r$ in the direction $\hat{\mathbf{r}}$ which is identified by the angle $\theta$ with respect to the stream-wise axis. The application of the ADF to experimental data requires the implementation of its two-dimensional surrogate, since the access to instantaneous particle positions is restricted to thin planes containing the mean flow. The operative definition for the two-dimensional version of the ADF reads:

$$
g(r, \theta)=\frac{\langle N(r, \theta) / \Delta S(r, \theta)\rangle}{N / A},
$$

where $N(r, \theta)$ represents the number of particles found in a small area $\Delta S=r \Delta r \Delta \theta$ centered in the direction $\theta$ at a distance $r$ away from the selected reference particle. In the above definition, $N / A$ represents the average particle concentration, while brackets denote a suitable ensemble average. Also, the separation $r$ parameterizes the inter-particle distance and the angle $\theta$ labels the spatial orientation of the particle pair. Figure 10 shows the quantity $g(r, \theta)$ computed for the numerical and the experimental dataset along two different directions, namely, for $\theta=45^{\circ}$ and 

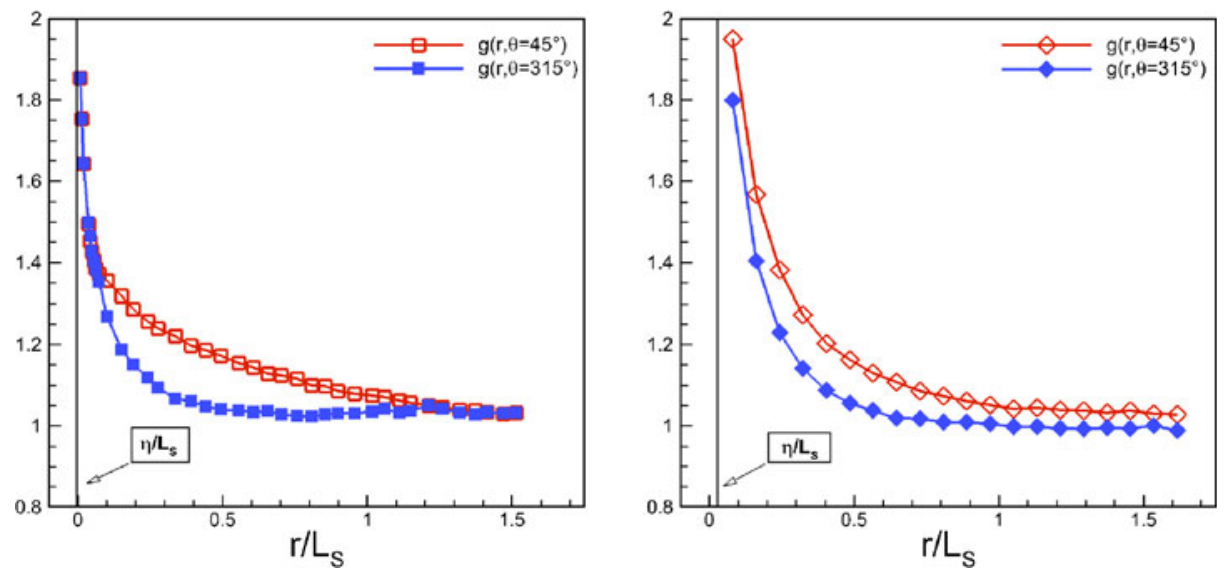

Fig. 10 The angular distribution function $g(r, \theta)$ computed along two orthogonal directions, respectively equal to $\theta \approx 45^{\circ}$ (red symbols) and $\theta \approx 315^{\circ}$ (blue symbols). The right and left panels correspond to the DNS (square symbols) and the experimental dataset (diamond), respectively

for $\theta=315^{\circ}$. Note that, since we are interested in the imprint of the mean shear, the pair separation $r$ is not rescaled as usual with the Kolmogorov length scale $\eta$ but is instead normalized by using the shear scale $L_{S}$. The rescaling with $L_{S}$ allows to examine particle transport in the range of scales which are affected by anisotropic advection for both the experimental and the numerical datasets, since $L_{S}$ identifies the lenghtscale above which turbulence is dominated by the mean shear (i.e. by energy production). Turbulence at scales below $L_{S}$ is instead driven by inertial energy transfer. The introduction of the shear scale is then the proper way to compare results from datasets at significantly different values of the Reynolds number. This is because the Reynolds number controls the extent of the range of scales below $L_{S}$, while the width of the production range above $L_{S}$ is fixed by the shear parameter $S^{*}$ that we are able to keep almost comparable between the two datasets.

Coming back to Fig. 10, we note that the phenomenon of preferential accumulation corresponds to the sharp increase of $g(r, \theta)$ as the scale separation $r$ is decreased for a fixed direction $\theta$. On the other hand, the large-scale anisotropy of the carrier field manifests itself via the dependence of the ADF on the direction $\theta$ (for fixed separation $r$ ). Specifically, the probability of finding particle pairs along the direction $\theta=45^{\circ}$ is systematically larger than the corresponding computed along any other direction (e.g., along $\theta=315^{\circ}$ ). This finding reflects the preferential alignment of particle clusters observed in the snapshots of Figs. 3 and 5 (left). The comparison of the ADF's depicted in the two panels of Fig. 10 indicates that the trend observed in the experiment is qualitatively well reproduced by the numerics, apart from some minor quantitative discrepancies. For instance, DNS results indicate that deviations from uniform concentration are confined to the smallest dissipative scales, while a wider range of separations is involved in the experiments. Possible reasons for this difference are the slight polydispersity of the particle population used in the experiment, as well as finite-size effects of the particles which are not 
accounted for by the numerics. The scaling behavior of the ADF highlights an important characteristic of the dispersion process of the solid phase [6]. Namely, the anisotropy of the particle field, as qualitatively conveyed by the differences between $g\left(r, 45^{\circ}\right)$ and $g\left(r, 315^{\circ}\right)$, is observed to be significant even at separations between the Kolmogorov scale $\eta$ and the shear scale $L_{S}$. As explained later in more detail, this behavior is in contrast with the properties of the carrier turbulence, where the anisotropy of the velocity field builds up in the range of scales between the integral and the shear scales, and falls off rapidly below $L_{S}$ in accordance with the Kolmogorov phenomenology of isotropy recovery [17]. On a more quantitative basis, the residual anisotropic structure of the particle clusters can be characterized by considering suitable decompositions of the ADF which are aimed at discriminating the isotropic component of the particle field and the anisotropic contributions. In three-dimensions, the general tool is the so-called $\mathrm{SO}(3)$ decomposition (see e.g. Ref. [18] and works cited therein). The technique allows to decompose a generic scalar statistical observable which depend on a separation vector $\mathbf{r}$, say $g(\mathbf{r})$, into components with a well-defined behavior under rotations. The basis functions used in the $\mathrm{SO}(3)$ decomposition are the spherical harmonics, and the decomposition for a scalar function reads:

$$
g(\mathbf{r})=\sum_{j=0}^{+\infty} \sum_{l=-j}^{l=+j} g_{j l}(r) Y_{j l}(\hat{\mathbf{r}}) .
$$

In this expression, the spherical harmonics $Y_{j l}(\hat{\mathbf{r}})$ are function of the unit vector $\hat{\mathbf{r}}=\mathbf{r} / r$ and account for the direction in which the observable $g(\mathbf{r})$ is being probed, while the coefficients $g_{j l}$ scale with the magnitude of the separation $r$ and encode the properties of the (particle) field at changing the observation scale. Note that the index $j$ measures the rate of change of the spherical harmonics over the unit sphere, and is therefore representative of the level of anisotropy of the observable. In particular, the isotropic component of the field corresponds to the index $j=0$, since the related spherical harmonics $Y_{00}$ is constant, while anisotropic contributions correspond to strictly positive values of $j$. In the present situation the $\mathrm{SO}(3)$ decomposition will be used for characterizing the fluid velocity field. Concerning the particle pair correlation function, since data are available only in a 2D domain, the relevant information concerning anisotropy can be obtained by using the simpler two-dimensional analogy, namely, the $\mathrm{SO}(2)$ decomposition. In this case, eigenfunctions of the group of 2D rotations, (i.e. sinusoidal functions) are used, see e.g. Ref. [19]. The SO(2) decomposition for the ADF is then simply given by:

$$
g(r, \theta)=\sum_{j} A_{j}(r) \cos (j \theta)+B_{j}(r) \sin (j \theta),
$$

The index $j$ again identifies subspaces with increasing levels of anisotropy. The isotropic projection $(j=0)$ is obtained by averaging the complete ADF along all directions:

$$
g_{0}(r)=\frac{1}{2 \pi} \int_{0}^{2 \pi} g(r, \theta) d \theta
$$

and corresponds to the classical RDF which characterizes the particle field in terms of the sole separation $r$ in isotropic turbulence. In order to quantify the anisotropy 

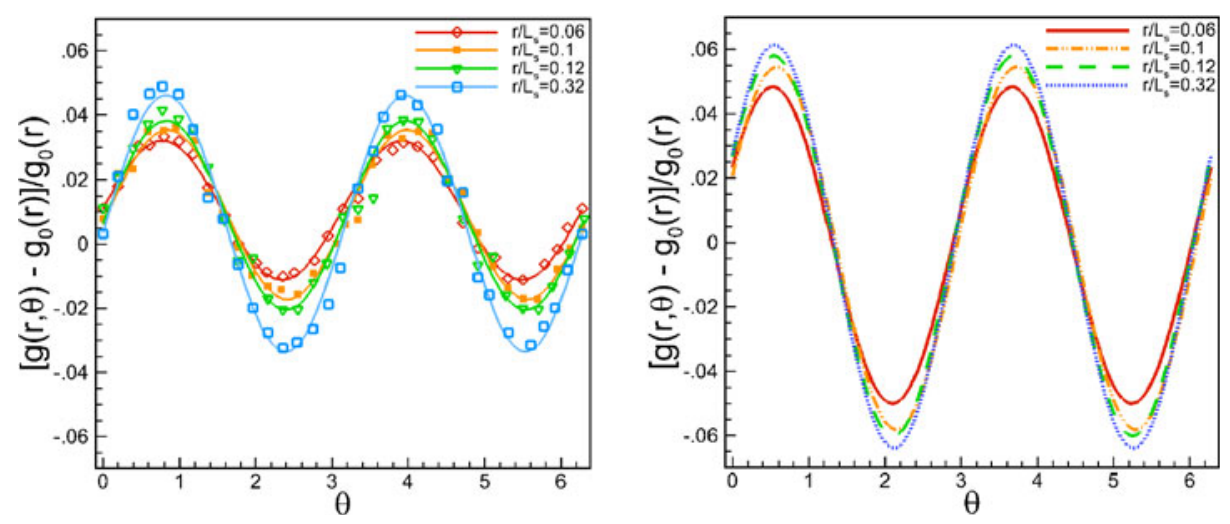

Fig. 11 The anisotropic component of the angular distribution function $g(r, \theta)$ computed at corresponding normalized separations $r / L_{S}$ for both the experimental (left panel) and DNS dataset (right panel)

of the clusters, we plot in Fig. 11 the ratio $\left[g(r, \theta)-g_{0}(r)\right] / g_{0}(r)$, which extracts the normalized anisotropic component of the ADF for both the experimental and the numerical data (left and right panels, respectively). In the plot, the non-dimensional separation $r / L_{s}$ is kept constant, and the quantity is displayed as a function of the angle $\theta$. The departure from isotropy appears in both datasets as a strong dependence of the observable on the direction $\theta$ : sharp maxima are observed at $\theta=45^{\circ}$, i.e. in the direction of the maximum mean strain, while minima are detected along the direction of maximum compression $\left(\theta=315^{\circ}\right)$. Note that similar levels of anisotropy are found in the two datasets at matching values of the dimensionless separation (slight differences in the intensity of the anisotropic signals are probably due to the small mismatch in shear- and particle parameters).

The anisotropy of the particle field is characterized in a slightly different way in Fig. 12 (left panel), where we plot the ratio $g_{2}(r) / g_{0}(r)$ which compares at each scale $r$ the most energetic anisotropic component of the ADF, namely $g_{2}(r)$ with the corresponding isotropic projection $g_{0}(r)$. Note that the separation is again made dimensionless by means of the shear scale in order to achieve a meaningful comparison between experimental and numerical data. The representation in Fig. 12 (left) allows to test in a more direct way the range of scales of the particle field where the directionality imprinted by the mean shear is still significant even in the range of scales where the fluid velocity fluctuations are almost isotropic, i.e., the peak anisotropy is reached well below the shear scale.

In order to better highlight the different structural properties of the fluid and particle phases, the anisotropy of the carrier velocity field can be computed and compared at each scale separation with the anisotropy of the particle field. The velocity field is described statistically in terms of the longitudinal structure function of order two $\left(S^{2}(r)\right)$, which is defined as:

$$
S^{2}(r, \hat{\mathbf{r}})=\left\langle([\mathbf{u}(\mathbf{x}+\mathbf{r})-\mathbf{u}(\mathbf{x})] \cdot \hat{\mathbf{r}})^{2}\right\rangle .
$$

The $\mathrm{SO}(3)$ decomposition defined in (Eq. 3) allows to extract both the isotropic $(j=0)$ and the anisotropic $(j>0)$ components of the above quantity. Similar to the 

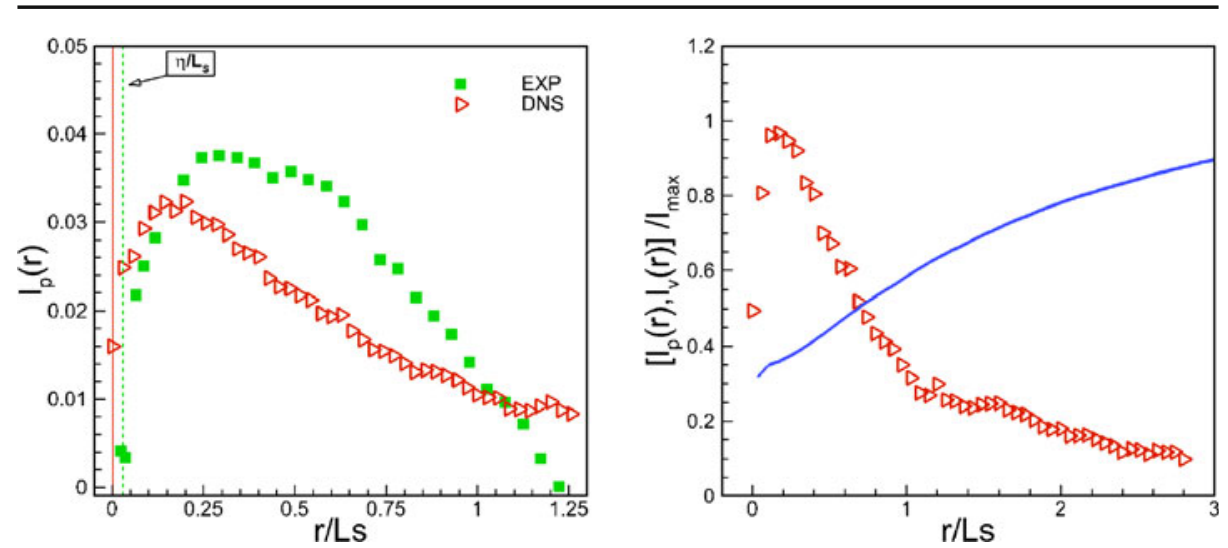

Fig. 12 Left panel: the anisotropy indicator $\left(g_{2}(r) / g_{0}(r)\right)$, i.e. the ratio of the most energetic anisotropic component of $g(r, \theta)$ to the isotropic projection, plotted versus non-dimensional particle pair separation $r / L_{S}$ for the two datasets. Right panel: the anisotropy indicator of the particle field $\left(g_{2}(r) / g_{0}(r)\right.$, symbols) compared with the anisotropy indicator of the fluid velocity field $\left(S_{2-2}^{2} / S_{00}^{2}\right.$, line), as computed from DNS data

particle field, the anisotropy of the velocity fluctuations can be gauged by comparing at each scale $r$ the magnitude of the anisotropic and the isotropic projections of $S^{2}$ with that of the , according to the indicator $I_{V}(r)$ defined as:

$$
I_{V}(r)=\frac{\sqrt{\sum_{j, l} S_{j l}^{2}(r)}}{\sqrt{S_{00}^{2}(r)}} \simeq \frac{S_{2-2}^{2}}{S_{00}^{2}} .
$$

The comparison between the anisotropy of the particle field and the anisotropy of the velocity field is shown in the right panel of the Fig. 12, where the appropriate indicators are displayed as a function of the non-dimensional scale $r / L_{S}$. The figure evidences that, contrary to the velocity field, the directionality of the particle statistics attenuates only at scales which are significantly smaller than the shear scale $L_{S}$. Namely, due to different inertia of the two phase the recovery of isotropy of the particle field is delayed as compared to the fluid [6].

As a final remark, it is also interesting to note that the level of anisotropy drops to negligible values as the pair separation goes to zero in the experimental data (Fig. 12 left), while a substantial residual anisotropy is observed at dissipative scales in the numerical data. This behavior is indeed not surprising once we recognize the different extent of the range of scales available for the isotropization process in the two cases. In fact, since the value of the non-dimensional shear intensity $S^{*}$ is similar in the experiment and in the numerics, the interval of isotropy recovery (between the shear and the Kolmogorov scales) is in fact controlled by the Reynolds number. The much larger value of $R e_{\lambda}$ in the experimental dataset allows for a larger separation between $L_{S}$ and $\eta$ and for a more effective isotropization of the velocity fluctuations which, in turns, results in an more isotropic advection of the particles. On the contrary, the relatively small Reynolds number typical of DNS simulations implies that the inertial range below the shear scale is too narrow to allow a significant isotropization of the clusters. In other words, closely below the 
shear scale viscous effects may become predominant with respect to inertial transfer making the isotropy recovery less effective.

\subsection{Particle clustering and large scale inhomogeneities}

In their simplest form, the radial distribution function, as well as its anisotropic counterpart (the ADF), provides a direct measure of the fluctuations of the particles field at scale $r$. A shortcoming of this observable is their inability to discriminate between inhomogeneities in the instantaneous concentration field which are induced by the mechanisms of preferential accumulation which prevail at small scales and inhomogeneities which are instead associated to a non uniform insemination of the incoming flow at relatively large scales. This point needs to be carefully addressed (particularly in experimental studies) to correctly quantify the relevance of preferential accumulation and obtain a sound comparison with numerical results.

To gain insight into the above issue, we have conducted some exploratory tests. Inspired by Ref. [20], we have considered the observable $\left[g_{0}(r)-1\right]$ (see e.g. [21]) which vanishes in a spatially homogeneous ensemble. The quantity $\left[g_{0}(r)-1\right]$ has been computed for a number of experimental datasets collected at different distances from the particle feeding device e.g. at $\Delta x \approx 0.5 \mathrm{~m}$ and at $\Delta x \approx 4.5 \mathrm{~m}$. At the closest measurement station, green symbols in the left panel of Fig. 13, the curve exhibits a much slower decay at the largest scales. Moreover, in the intermediate range of scales, say $r / L_{S} \simeq 0.5 \div 1.5$, the data indicate a significant increase of the number of particles pairs found at that separations. However, this may be considered as a "fictitious" clustering, which represents in reality the signature of insufficient mixing of the particle "cloud" which are injected just upstream of the measurement station. Actually at $\Delta x \approx 4.5 \mathrm{~m}$ downstream from seeding devices the "cloud" of particles has been exposed for a much longer time to turbulent mixing, resulting in a smearing out of the large scales inhomogeneities which are unavoidably introduced by the seeding process. In this case, actually, the observable $\left[g_{0}(r)-1\right]$ quickly approaches zero at the largest scales. Moreover, in the intermediate range of scales $r / L_{S} \in[0.5: 1.5]$, the data show only a weak increase as the scale separation is reduced, and eventually increase dramatically as the smallest scales are reached (denoting the occurrence of clustering). This different behavior can be understood in the light of recent findings for isotropic turbulence described in Ref. [20]. The authors of this work suggest that the slow decay of $\left[g_{0}(r)-1\right]$ at large inertial scales could be modelled in terms of a scaling law derived from simple dimensional arguments in the context of the Kolmogorov phenomenology. Actually, when an initially spatially non-uniform passive scalar field is mixed by turbulence, the magnitude of the pair correlation function is controlled by the integral scale $L_{0}$ and is characterized by a scaling law behavior in the form: $\left[g_{0}(r)-1\right] \sim 1-\left(r / L_{o}\right)^{2 / 3}$, see e.g. [22]. This scaling is reported for comparison in the right panel of Fig. 13 for the data measured at $\Delta x \approx 0.5 \mathrm{~m}$ and at $\Delta x \approx 4.5 \mathrm{~m}$ (note that the shear scale is used in this case to mark the border of the inertial range). The agreement with the particle statistics at the closest station is indeed remarkable, confirming that the particle-laden flow has not yet reached a fully homogeneous state. Farther away, the argument based on the turbulent mixing is not able to describe the variation of the particle correlation any more (in this case, the particle distribution can be described by another functional form, see Ref. [6]). 

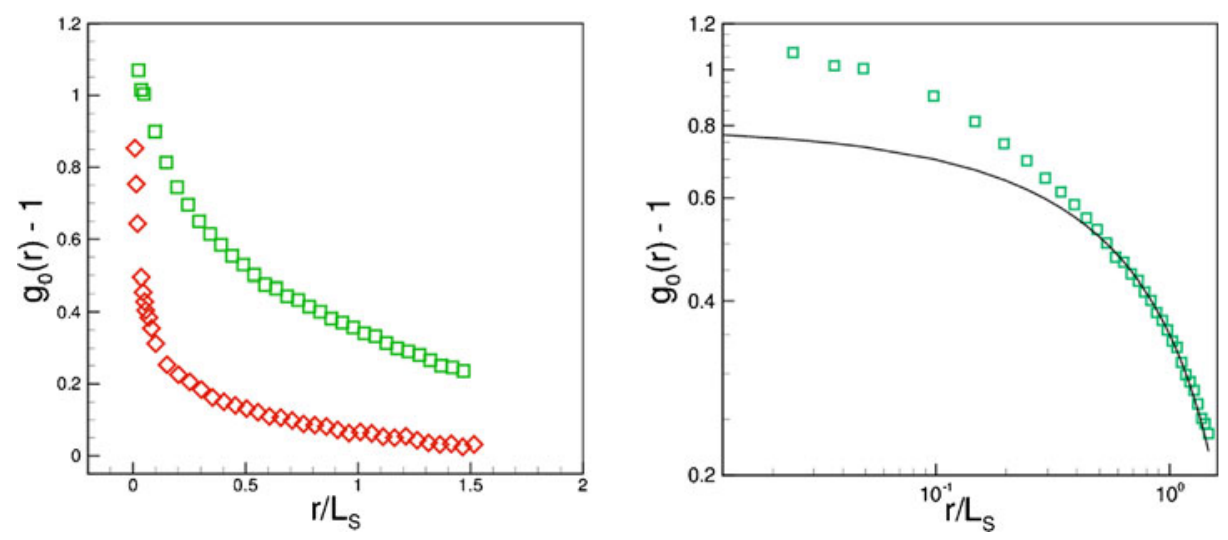

Fig. 13 Left panel: the quantity $[g(r)-1]$ measured at different distances from the curved screen. Green squares correspond to data collected near the seeding device $(\Delta x \approx 0.5 \mathrm{~m})$, red diamonds represent data collected far downstream $(\Delta x \approx 4.5 \mathrm{~m})$. Right panel: the quantity $[g(r)-1]$ measured at $\Delta x \approx 0.5 \mathrm{~m}$ is tested against the prediction $1-\left(r / L_{S}\right)^{2 / 3}$ (line) suggested in Ref. [20]

In order to have further insight into the crosstalk between effects due to largescale nonuniformities and turbulent clustering, we have designed a targeted numerical numerical experiment. We considered a time-evolving homogeneous shear flow, i.e. a configuration which can be easily recasted into a spatially evolving flow by means of the transformation $x^{*}=S t$, where $x^{*}$ is the streamwise coordinate made dimensionless with the vertical dimension $L_{y}$ of the experimental apparatus. The flow was initially seeded with Lagrangian tracers and inertial particles distributed at random homogeneous positions in a sphere of radius $\pi / 2$. The initial condition is homogeneous at small scales (i.e., for separations smaller than the sphere diameter), while it is strongly non homogeneous at larger scales. Figure 14 reports the observable $\left[g_{0}(r)-1\right]$ at different simulation time steps (corresponding to different downstream locations in the experiment) for both the Lagrangian tracers -linesand the inertial particles -symbols. The data show distinct behaviors of the pair correlation function at small and large scales respectively. At small scales, $\left[g_{0}(r)-1\right]$ rapidly diverges, indicating the occurrence of clustering. On the contrary, when large separations are probed, the pair correlation function is strongly affected by the large scale non homogeneous effects inherited from the initial condition. In fact, as previously discussed, the quantity $\left[g_{0}(r)-1\right]$ is characterized by a slow decaying tail at the largest scales, which typifies spatially non homogeneous distributions of a passive scalar. The data also convey an other important massage: during the early stages of the simulation, i.e. close to the particle injection point in the experiment, the behavior of Lagrangian tracers and of inertial particles does not substantially differ. Hence, we are confident that the scaling law postulated in Ref. [20] may be representative of large scales inhomogeneities also for the inertial particles, as discussed above. The fact that inertial particles with $S t=0.3$ behave essentially as the Lagrangian tracers, as shown by symbols and lines in Fig. 14, during the initial stages of the simulation is not surprising. In fact, the response of inertial particles to turbulent fluctuations is parameterized in terms of the Stokes number $S t=\tau_{p} / \tau_{f}$, where $\tau_{f}$ is a characteristic timescale of the turbulent fluctuations. During 
Fig. 14 Pair correlation function computed for both Lagrangian tracers (lines) and inertial particles (symbols), for different nondimensional times $S * t$ along the simulation

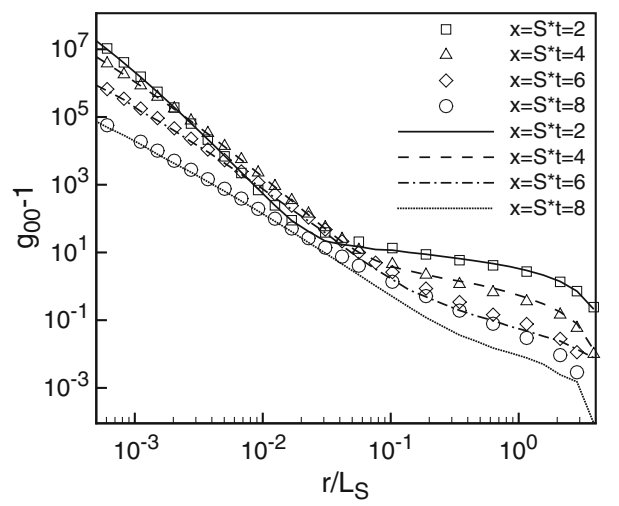

the early stages of the dispersion process, particles are essentially dragged by the most energetic (large-scale) turbulent motions, which are characterized by a time scale comparable with the integral eddy turn-over time $\tau_{0}$, i.e. $\tau_{f} \simeq \tau_{0}$. The relevant Stokes number is then $S t_{0}=\tau_{p} / \tau_{0} \rightarrow 0$, which suggests that the response of inertial particles to the highly energetic large scale eddies should not differ significantly from the response of a passive tracer.

\section{Concluding Remarks}

The spatial distribution of heavy particles $(S t \approx 0.3 \div 0.5)$ advected by a homogeneous shear flow in the one-way coupling regime has been characterized by means of a combination of experimental and numerical investigations. The emphasis is placed on the aspects of preferential accumulation and on the anisotropic features of the particle field. The visual inspection of particle snapshots indicates that particles tend to aggregate into thin clusters outside vortex cores, and that the mean velocity gradient induces an evident preferential orientation on the particle clusters. An analysis conducted in terms of Voronoï tessellations provides a more quantitative characterization of the clustering process. In particular, the analysis of Voronoï diagrams has shown that specific features observed in isotropic conditions, e.g. the selfsimilar behavior of small-scale clustering regions, are also shared by homogeneous shear turbulence. Overall, an extremely satisfactory agreement between numerical and experimental findings was observed.

The use of the Angular Distribution Function provides an alternative way to characterize the geometry of the clusters, and in particular to estimate the anisotropy content of the particle field at changing the scale of observation. The main effect associated with particle inertia consists in a delay of the isotropy recovery process of the particle concentration field as compared to the underlying velocity field. Despite the differences of the flow parameters, the numerical approach was again found to correctly reproduce the qualitative variation of the anisotropy level measured experimentally. The above correspondence among the obtained results is instrumental for further comparisons, namely for cases like the two way coupling regime, where an appropriate modelling of the inter-phase momentum exchange is required in the numerical simulation. 


\section{References}

1. Balachandar, S., Eaton, J.K.: Turbulent dispersed multiphase flow. Ann. Rev. Fluid Mech. 42, 111-133 (2010)

2. Jacob, B., Olivieri, A., Miozzi, M., Campana, E.F., Piva, R.: Drag reduction by microbubbles in a turbulent boundary layer. Phys. Fluids 22, 115104 (2010)

3. Gualtieri, P., Picano, F., Sardina, G., Casciola, C.M.: Clustering and turbulence modulation in particle laden shear flows. J. Fluid Mech. 715, 134-162 (2013)

4. Dunn, W., Tavoularis, S.: The use of curved screens for generating uniform shear at low Reynolds numbers. Exp. Fluids 42, 281-290 (2007)

5. Corrsin, S.: Local isotropy in turbulent shear flow. Tech. Rep. (NACA RM58B11, 1958) (2007)

6. Nicolai, C., Jacob, B., Piva, R.: On the spatial distribution of small heavy particles in homogeneous shear turbulence. Phys. Fluids 25, 083301 (2013)

7. Prosperetti, A., Tryggvason, G. (eds.): Computational Methods for Multiphase Flow. Cambridge University Press (2007)

8. Gualtieri, P., Picano, F., Casciola, C.M.: Anisotropic clustering of inertial particles in homogeneous shear flow. J. Fluid Mech. 629, 25-39 (2009)

9. Monchaux, R., Bourgoin, M., Cartellier, A.: Analyzing preferential concentration and clustering of inertial particles in turbulence. Int. J. Multiphase Flow 40, 1-18 (2012)

10. Monchaux, R., Bourgoin, M., Cartellier, A.: Preferential concentration of heavy particles: a Voronoï analysis. Phys. Fluids 22, 113304 (2010)

11. Ferenc, J.-S., Néda, Z.: On the size distribution of Poisson Voronoí cells. Phys. A 385, 518 (2007)

12. Monchaux, R.: Measuring concentration with Voronoï diagrams: the study of possible biases. New J. Phys. 14(9), 095013 (2012)

13. Meneguz, E., Reeks, M.W.: Statistical properties of particle segregation in homogeneous isotropic turbulence. J. Fluid Mech. 686, 338 (2011)

14. Ijzermans, R., Meneguz, E., Reeks, M.W.: Segregation of particles in incompressible random flows: singularities, intermittency and random uncorrelated motion. J. Fluid Mech. 653, 99 (2010)

15. Sundaram, S., Collins, L.R.: Collision statistics in an isotropic particle-laden turbulent suspension. Part 1. Direct numerical simulations. J. Fluid Mech. 335, 75-109 (1997)

16. Reade, W.C., Collins, L.R.: Effect of preferential concentration on turbulent collision rates. Phys. Fluids 12, 10 (2000)

17. Casciola, C.M., Gualtieri, P., Jacob, B., Piva, R.: Scaling properties in the production range of shear dominated flows. Phys. Rev. Lett. 95, 024503 (2005)

18. Biferale, L., Procaccia, I.: Anisotropy in turbulent flows and in turbulent transport. Phys. Rep. 414, 43-164 (2005)

19. Celani, A., Lanotte, A., Mazzino, A., Vergassola, M.: Fronts in passive scalar turbulence. Phys. Fluids 13, 1768 (2001)

20. Saw, E.W., Shaw, R.A., Ayyalasomayajula, S., Chuang, P.Y., Gylfason, A.: Inertial clustering of particles in high Reynolds number turbulence. Phys. Rev. Lett. 100, 214501 (2008)

21. Collins, L.R., Keswani A.: Reynolds number scaling of particle clustering in turbulent aerosols. New J. Phys. 6(1), 119 (2004)

22. Lumley, J.L., Panofsky, H.A.: The Structure of Atmospheric Turbulence. John Wiley \& Sons, New York (1964) 